Case Report

\title{
Hyperthyroid Fetal Goiter: A Case Report
}

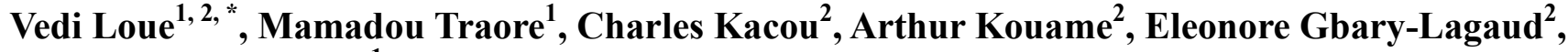 Julie Grevoul-Fesquet ${ }^{1}$}

\author{
${ }^{1}$ Sud Francilien Hospital Center (Woman-Mother-Child Pole), Paris Descartes University, Paris, France \\ ${ }^{2}$ Faculty of Medical Sciences (Mother and Child Department, University Hospital of Cocody), Felix Houphouet-Boigny University, Abidjan, \\ Cote d'Ivoire
}

Email address:

drlouevedi@yahoo.fr (V. Loue)

${ }^{*}$ Corresponding author

\section{To cite this article:}

Vedi Loue, Mamadou Traore, Charles Kacou, Arthur Kouame, Eleonore Gbary-Lagaud, Julie Grevoul-Fesquet. Hyperthyroid Fetal Goiter: A Case Report. Journal of Gynecology and Obstetrics. Vol. 7, No. 3, 2019, pp. 76-80. doi: 10.11648/j.jgo.20190703.14

Received: December 5, 2018; Accepted: February 1, 2019; Published: June 11, 2019

\begin{abstract}
The persistent presence of maternal thyroid-stimulating hormone receptor antibody in patients with Graves'disease could cause fetal hyperthyroid goiter during pregnancy. The recognition and treatment of hyperthyroid fetal goiter are believed to be very important to optimize growth and intellectual development in affected fetuses. We report here, a case of a hyperthyroid fetal goiter identified by ultrasound exam at 27 weeks 5 days of gestation in a women having Graves' disease and on antithyroid drugs therapy. This observation shows the fetal risk associated with the persistence of anti-TSH receptor antibodies in mothers with Graves' disease and the possibility of fetal approach.
\end{abstract}

Keywords: Graves' Disease, Thyroid Hormones, Fetal Hyperthyroid Goiter, Prenatal Diagnosis

\section{Introduction}

Fetal goiter, which signals a thyroid dysfunction, is a rare condition. It can be caused by several different pathologies: including dysgenesis, dyshormonogenesis, maternal ingestion of antithyroid drugs, transplacental passage of maternal antibodies, thyroid stimulating hormone receptor mutation, or tumors [1]. Among fetal goiter, fetal hyperthyroidism goiter is more rare with an unknown incidence than the goitrous hypothyroidism which incidence is $1 / 30000-50000$ live births [2]. Fetal hyperthyroid goiter can cause maternal, obstetric and fetal complications and has fetal mortality rate of $16 \%$ to $25 \%$ if untreated, requiring early diagnosis and appropriate treatment to restore euthyroidism [3-4]. The persistent presence of maternal thyroid-stimulating hormone receptor antibody (Trab) in patients with Graves' disease could cause fetal hyperthyroid goiter during pregnancy by stimulating the thyroid gland, leading to excessive thyroid hormone secretion. The risk correlates with the TRab titer [2]. We report here, a case of a hyperthyroid fetal goiter identified by ultrasound exam at 27 weeks 5 days of gestation in a women having
Graves' disease and on antithyroid drugs therapy. Through this case, we recall the physiopathological, diagnostic and therapeutic aspects of this pathology.

\section{Case Presentation}

The patient, D. G. T, a 32 years-old woman of Malian origin, seventh gravida fifth para was followed up at Sud Francilien Hospital Center (Corbeil-Essonnes, France) since the beginning of her 7th pregnancy. In her obstetric history, she had 5 live and normal children. The couple was not consanguineous and the risk of trisomy-21 was $1 / 10,000$ in this patient. She also had Graves' disease discovered and treated since 2006 intermittently by Propylthiouracil* $100 \mathrm{mg}$ per day. She presented a recurrence in 2016 during her seventh pregnancy with cardiovascular signs (hypertension, palpitations, tremor and tachycardia).

The dating ultrasound at 12 weeks of amenorrhea did not reveal morphological abnormalities, the ultrasound of the maternal thyroid was normal. Blood levels of maternal thyroid hormones revealed: free triiodothyronine (fT3): $15.4 \mathrm{ng} / \mathrm{L}$ $(3.1<\mathrm{N}<6.8)$; free thyroxine (fT4): 46,5ng/L $(9.5<\mathrm{N}<18)$; 
thyroid stimulating hormone (TSH): $0.01 \mathrm{mUI} / \mathrm{L}$ $(0.27<\mathrm{N}<4.2)$; thyrotropine receptor autoantibodies (TRAb): 16,6 (normal value $<1 \mathrm{UI} / \mathrm{L}$ ). Maternal hyperthyroidism is then concluded in response to the high level of thyroid hormones with low TSH level. The treatment of fetal hyperthyroidism was performed initially by giving $150 \mathrm{mg} /$ day of Propylthiouracil (PTU) to the mother. This dose was gradually increased up to $300 \mathrm{mg} /$ day at the end of pregnancy, based on ultrasound monitoring. She was also on beta-blocker (Avlocardyl * $160 \mathrm{mg}$ per day).

The obstetric ultrasound of 22 weeks of gestation was not done because the patient did not come to her appointment, however an ultrasound performed at 27 weeks 5 days revealed a fetal goiter with an enlarged thyroid size greater than the 95th percentile measuring $24.97 \times 16.21 \mathrm{~mm}$, circumference: $65.42 \mathrm{~mm}$ with central hypervascularization (figures 1 and 2).

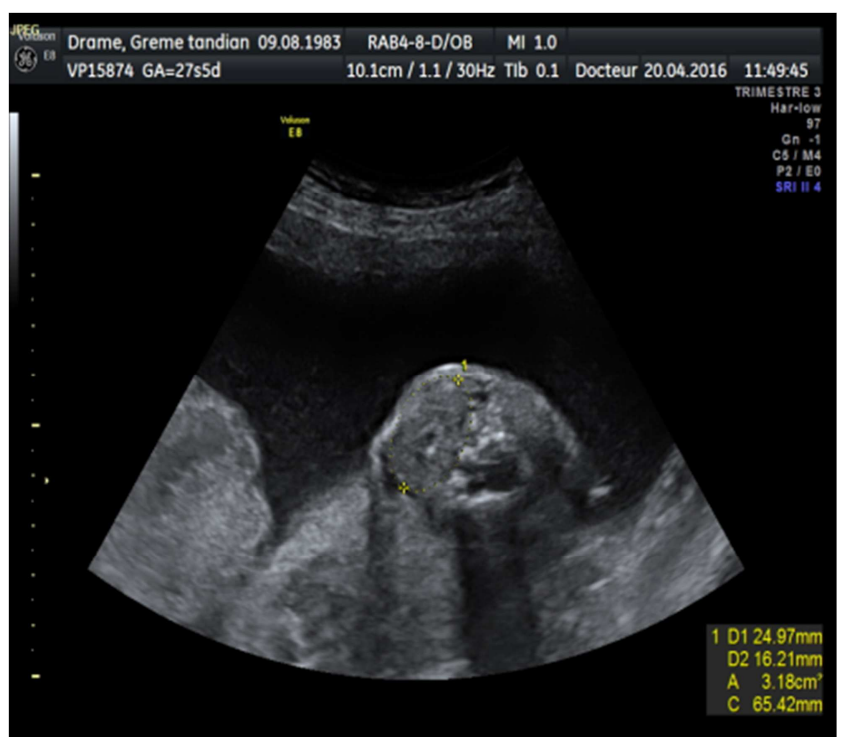

Figure 1. Ultrasound examination performed at 27 week +5 days of gestation showing an enlarged thyroid fetal gland measuring $24.97 \times 16.21 \mathrm{~mm}$; circonference: $65.42 \mathrm{~mm}$.

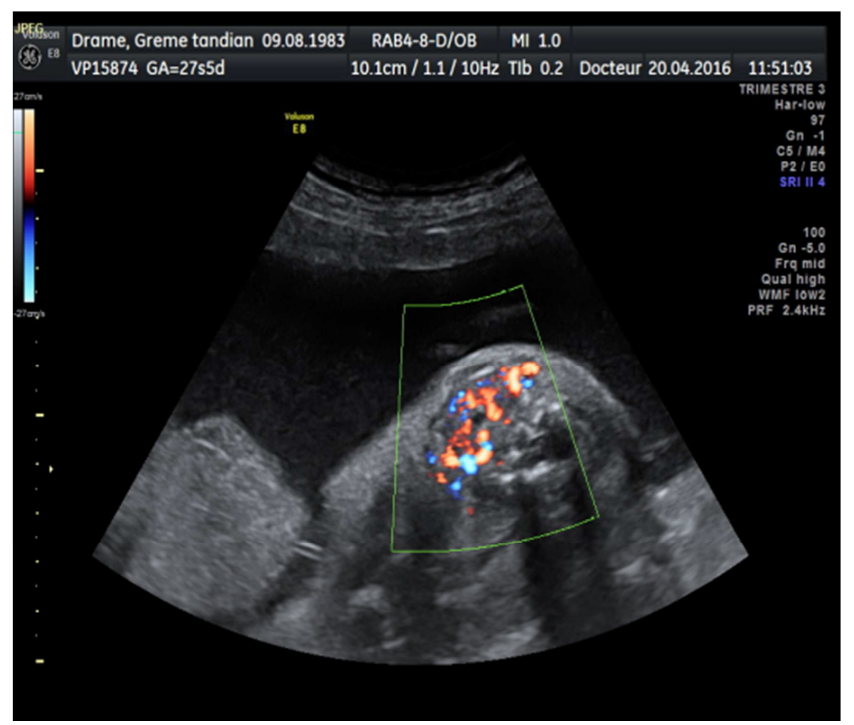

Figure 2. Color Doppler image of fetal neck showing central or global vascularization of a goiter.
There were also a slight excess of amniotic fluid (large cistern: $75 \mathrm{~mm}$ ), normal umbilical Doppler, normal morphology, good movements, satisfactory growth to 50th percentile and fetal permanent tachycardia. Maternal biological control showed: fT4: $29.2 \mathrm{ng} / \mathrm{ml}$; fT3: $8.9 \mathrm{ng} / \mathrm{ml}$; TSH: $<0.01 \mathrm{mUI} / \mathrm{L}$. The medical file was presented to the Multidisciplinary Center for Prenatal Diagnosis and an ultrasound carried out by a referent confirmed the fetal goiter. The diagnosis of hyperthyroid fetal goiter due to transplacental passage of antibodies against the thyroid-stimulating hormone (TSH) receptor was retained in front of maternal history (Graves'disease), maternal biology (high level of TRAb) and obstetric ultrasound results (fetal goiter with central hypervascularization and tachycardia). It has been then recommended to continue Propyl-thiouracil and ultrasound monitoring was recommended every two weeks with dosage of maternal thyroid hormones per week. This closed monitoring revealed a gradual decrease in the size of fetal thyroid with full normalization at 31 weeks. At term, on ultrasound performed the 13/07/16 at 38 weeks 3 days, the fetus was eutrophic with good vitality. Fetal heart rate, amniotic fluid and fetal thyroid size were all normal. The patient hormones levels were also normal without clinical signs. The birth occurred at 38 weeks 5 days of gestation spontaneously per vaginal delivery. The newborn male, weighing: $2900 \mathrm{~g}$, cranial perimeter: $34 \mathrm{~cm}$, size: $50 \mathrm{~cm}$, Apgar score: 10/10. The newborn was transferred to pediatrics for specialized management. Neonatal clinical examination was normal without palpable goiter or clinical signs of hyperthyroidism. The newborn's thyroid hormone levels were normal up to 10 weeks of life. Since then, the volume and the thyroid function of the child have remained normal.

\section{Discussion}

\subsection{Physiopathology}

Fetal hyperthyroidism may be the consequence of two distinct pathologies. The first is of autoimmune origin, it is Graves' disease. The second, which is very rare, is of genetic origin and is linked either to the mutation of the TSH receptor or to an activating mutation of Guanine Nucleotide binding protein Alpha Stimulating (GNAS) gene: this gene encodes the alpha subunit of the $\mathrm{G}$ protein coupled to adenylate cyclase, whose role is to induce the synthesis of thyroid hormones [5].

Graves' disease is the most common cause of hyperthyroidism. It is an autoimmune thyroiditis in which maternal autoantibodies stimulate the overproduction of thyroid hormones by the thyroid follicular cells, thus inducing a state of hyperthyroidism. These immunoglobulins, IgG type, have two types of possible action: some stimulate the synthesis of Cyclic Adenosine Monophosphate by these cells, these are the thyroid stimulating immunoglobulins (TSI) while other antibodies will block the binding of TSH with its receptor, these are the thyroid binding inhibiting Immunoglobulins (TBII). Among pregnant women with Graves' disease, $0.2 \%$ develops clinical hyperthyroidism and, 
among them, 1 to $5 \%$ of their newborns will present a neonatal hyperthyroidism due to transplacental passage of TRAb $[2,6]$. Indeed, the fetal thyroid starts its synthesis of thyroid hormone from the 12th week of gestation. Before that date, the fetus is therefore entirely dependent on maternal thyroid hormones. The thyrotropine receptors, meanwhile, will be sensitive to the fetal TSH (maternal TSH does not cross the placenta) from the second trimester of pregnancy. However, it is also at this time that the transplacental passage of maternal autoantibodies increases. Therefore, these autoantibodies can focus on the fetus TSH-receptors and induce an excessive biosynthesis of hormones responsible for fetal hyperthyroidism. This explains why fetal hyperthyroidism doesn't appear until the second half of pregnancy, and also in patients with a rate of higher autoantibodies (TRAb) [2, 5, 7-8]. The fetal risk concerns both the fetuses of mothers with the past or current Graves' disease and includes: intrauterine growth retardation, prematurity, intrauterine fetal death $(15-25 \%$ if untreated), fetal Graves' disease [2-3, 6]. Moreover the presence of autoantibodies can encourage the development of fetal goiter, responsible in rare cases to a compression of the trachea or fetal esophageal [6]. Fetal hypothyroidism induced due to synthetic antithyroid drugs by transplacental passage may be accompanied by goiter but inconstantly [9]. These different pathophysiological mechanisms clearly understand the occurrence of hyperthyroid fetal goiter in this pregnant patient treated for Graves' disease by PTU.

\subsection{Diagnosis}

The measurement of thyroid and screening of fetal goiter can be reliably to 20 weeks of pregnancy, especially when it comes to fetus at risk, especially among mothers of Graves' disease treated with antithyroid drugs, or in patients who have Graves disease with TRAb presence as in the present case, and also in patients treated of their Graves disease (surgery or radioiodine) but keeping positive TRAb [6-7].

In this present case, fetal goiter was diagnosed at 27 weeks 5 days of gestation. Ultrasound of 22 weeks could not be performed because of the non respect of the appointment by the patient. The ultrasound diagnosis of goiter (sensitivity $100 \%$, specificity $92 \%$ ) is generally quite easy [7]. The thyroid gland is a homogeneous solid mass identified at the anterior of the fetal neck, bilobed, limited laterally by the vascular structures and back by the trachea [2]. However, first eliminate all differential diagnosis of neck mass [6]. Fetal goiter was defined as a thyroid circumference or diameter $>95^{\text {th }}$ percentile for gestational age [10-11]. When fetal goiter was found, color flow Doppler examination of the thyroid was performed with the velocity scale adjusted to $13 \mathrm{~cm} / \mathrm{s}$. Ultrasound revealed global or central vascularization, permanent fetal tachycardia (fetal heart rate continuously faster than $160 \mathrm{bpm}$ ), accelerated bone maturation, increased fetal movements [2]. Fetal hyperthyroidism can be suspected also when there is IUGR, sometimes accompanied by oligohydramnios (very low specificity) and other signs accompanying goiter are not discriminating such as polyhydramnios being related to the goiter volume rather than etiology. Even, heart failure is not unique to hyperthyroidism.
All these signs are inconstant, non-specific or observed late and are of no value individually [6]. However, several of these signs may be absent or difficult to interpret. In this case, the diagnostic suspicion rested on the discovery of a goiter on the ultrasound with a central vascularisation in the Doppler and fetal tachycardia. Apart from maternal history indicating the patient's history of Graves disease treated with antithyroid, maternal antithyroid hormones levels are essential and includes dosage of TSH, fT4, fT3 which revealed a low level of TSH and the high levels of fT4 and fT3. The immunological balance sheet: determination of antibody anti-thyroglobulin, antithyroid peroxydase and antibody anti-TSH receptors (TSI and TBII) that must be present. In practice, positivity of TRAb is sufficient to demonstrate that the patient is a carrier of such antibodies as revealed in our case. All these enabled the diagnosis of hyperthyroid fetal goiter by transplacental passage of antibodies anti-TSH receptors of stimulatory effect in this woman. In practice, the diagnosis of certainty is a direct diagnosis, based on fetal thyroid hormones levels (TSH, fT4, fT3) by fetal blood sampling (FBS), not to mention the possible risks of this invasive exam (infection, fetal bradycardia, iatrogenic prematurity) [2]. Fetal blood sampling by cordocentesis was not necessary in the case we present. Indeed Fetal blood sampling should be indicated for the presence of a goiter without that we can identify a state of fetal hyperthyroidism or hypothyroidism, for fetuses of severe risk of hypothyroidism and when intra-amniotic injection of thyroxine is indicated [12-13]. Fetal blood sampling may be proposed as a first-line treatment in the case of goiter occurring in the fetus of a mother without a thyroid antecedent, possibly in the case of a combination of stimulating antireceptor antibodies with a synthetic antithyroid treatment and, if despite the reduction of synthetic antithyroid there is a persistence of goiter. The intra-amniotic fetal thyroid hormones level could be an alternative to the FBS [6]. The maternal dosage of the compound $\mathrm{W}$ of placental origin could reflect fetal thyroid metabolism and constitute a new option diagnostic [11]. Some authors have used MRI to confirm the diagnosis; however the MRI does not seem at this time make any additional element in the characterization of fetal goiter [11].

\subsection{Treatment}

In practice, in the case of Graves' disease, follow-up should start at the beginning of pregnancy by assaying the levels of anti-TSH receptor antibodies, the persistence of which can lead to a fetal risk. Maternal hyperthyroidism and fetal hyperthyroidism themselves may induce fetal goiter. Monitoring of maternal thyroid function and good fetal development is required. This surveillance should be increased in the second part of the pregnancy from 20-22 SA, when fetal hyperthyroidism begins to manifest. Maternal real hyperthyroidism justifies management in order to limit risks for the mother (miscarriage, hypertension, pre-eclampsia, cardiac failure and placental abruptio) or the child (prematurity, intrauterine growth retardation, goiter, fetal hyperthyroidism and death). In short term, the risk is represented by cardiac insufficiency and in long term, by the 
risk of craniostenosis accompanied by delayed psychomotor development of the child [9]. As for maternal hyperthyroidism, the presence of a large goiter remains the main indication of an in utero management. Treatment aims to reduce the volume of goiter in order to avoid complications: reduce hydramnios (secondary to esophageal compression swallowing disorders) in order to avoid the risk of premature delivery, decrease cervical hyper-extension and the risk of dystocia at delivery, reduce tracheal compression to limit neonatal morbidity or even perpartum mortality by respiratory distress or asphyxia, and exceptionally, decrease the arteriovenous shunt and the risk of heart failure [2]. The major concern in the treatment of hyperthyroidism in pregnant women is to maintain a perfect hormonal balance in the mother while avoiding fetal hyperthyroidism or switching to hypothyroidism as a result of the placental passage of the molecules. Antithyroid dosages should be as low as possible during pregnancy to avoid fetal hypothyroidism, even if the mother is slightly hyperthyroid. In the first quarter: Propylthiouracil can be used as low as possible during the first trimester of pregnancy $[2,5]$. In the 2nd and / or 3rd trimester, due to the undesirable liver effects of the PTU, it is preferable, if possible, to relieve the PTU with Carbimazole beyond the 1 st trimester [4-5, 14]. If PTU is maintained, use the lowest possible dosage. The dose should be gradually decreased (so that the maternal fT4 level is at the upper limit of normal) and provide close ultrasound monitoring of the fetal thyroid to detect possible fetal hyperthyroidism transplacental passage of antibody or fetal goiter by transplacental passage of antithyroid $[1,3,6,8,11]$. If the treatment is continued until the birth, inform the maternity team so that it can adapt the reception of the newborn. Indeed, a review of neonatal thyroid function is desirable. In this case, the discovery of maternal hyperthyroidism required placing the patient under Propylthiouracil* $150 \mathrm{mg}$ per day at the beginning of pregnancy for 4 months followed by a gradual increased to stabilize the dose at $300 \mathrm{mg}$ per day. Beta blocker therapy has been initiated for symptomatic use. Although in the third trimester there was a spontaneous alteration of hyperthyroidism leading to a decrease in ATS, or even cessation if the mother was euthyroid, the termination in this case was linked to the ultrasound discovery of $27 \mathrm{SA}+5$ days of fetal goiter. Maternal biological monitoring every week and fetal ultrasound monitoring every two weeks were advocated. At $38 \mathrm{SA}+5$ days, the patient gives birth spontaneously vaginally of a normotrophic newborn. Given to pediatricians, his physical examination does not reveal goiter and his thyroid balance of TSH, fT4 and anti-TSH receptor antibodies in the first 48 hours, renewed on 14 th days later was normal and up to 10 weeks of life. Since then, the volume and the thyroid function of the child have remained normal.

\section{Conclusion}

A fetal hyperthyroid goiter is uncommon but potentially serious complication of maternal Grave' disease. This observation shows the fetal risk associated with the persistence of anti-TSH receptor antibodies in mothers with Graves' disease. It also shows the possibility of fetal approach to evaluate and normalize fetal thyroid function.

\section{Conflict of Interest}

All the authors do not have any possible conflicts of interest.

\section{Author's Contributions}

All authors were major contributors in writing the paper. All authors read and approved the final paper.

\section{Ethical Approval}

The study was approved by the Houphouët-Boigny's University and the Paris Descartes' University ethics committees.

\section{References}

[1] Aynadis Alemu, Betelihem Terefe, Molla Abebe, Belete Biadgo. Thyroid hormone dysfunction during pregnancy: A review. Int J Reprod BioMed 2016; 14, 11: 677-86.

[2] Kazakou P,Theodora M, Kanaka-Gantenbein C,et al. Fetal hyperthyroidism associated with maternal thyroid autoantibodies: A case report. Case Reports in Women's Health 2018; 19: e00081.

[3] Andersen SL, Laurberg P. Managing hyperthyroidism in pregnancy: current perspectives. International Journal of Women's Health 2016: 8 497-504.

[4] Kurtoğlu S, Özdemir A. Fetal neonatal hyperthyroidism: diagnostic and therapeutic approachment. Turk Pediatri Ars 2017; 52: 1-9.

[5] De Leo S, Lee SY, Braverman LE. Hyperthyroidism. Lancet 2016; 388 (10047): 906-918.

[6] Nguyen CT, Sasso EB, Barton L, Mestman JH. Graves' hyperthyroidism in pregnancy: a clinical review. Clinical Diabetes and Endocrinology 2018; 4:4.

[7] Bucci I, Giuliani C, Napolitano G. Thyroid-Stimulating Hormone Receptor Antibodies in Pregnancy: Clinical Relevance. Frontiers in Endocrinology 2017; 8: 137.

[8] Banige M, Estellat C, Biran V, et al. Study of the Factors Leading to Fetal and Neonatal Dysthyroidism in Children of Patients With Graves Disease. Journal of the Endocrine Society 2017; 1,6: 751-61.

[9] Léger J. Management of Fetal and Neonatal Graves' Disease. Horm Res Paediatr. 2017; 87 (1): 1-6.

[10] Taff C. Prenatal diagnosis and treatment of fetal goiter. $J$ Diagnosis Medical Sonography 2016; 32, 1: 40-3.

[11] Neto JFG, Junior EA, Costa JIF, et al. Fetal goiter conservatively monitored during the prenatal period associated with maternal and neonatal euthyroid status. Obstet Gynecol Sci 2016; 59, 1: 54-7. 
[12] Mastrolia SA, Mandola A, Mazor M, et al. Prenatal diagnosis and treatment perspective of fetal hypothyroidism with goiter. $J$ Matern Fetal Neonatal Med 2015; 28(18):2214-20.

[13] Min-Jung Kim, Yong-Hwa Chae, So-Young Park, Moon-Young Kim. Intra-amniotic thyroxine to treat fetal goiter. Obstet Gynecol Sci 2016;59(1):66-70.
[14] Song R, Lin H, Chen Y, Zhang X, Feng W. Effects of methimazole and propylthiouracil exposure during pregnancy on the risk of neonatal congenital malformations: A metaanalysis. PloS ONE 2017; 12(7): e0180108. 